\title{
The Antisense long noncoding RNA AGAP2-AS1 regulates cell proliferation and metastasis in Epithelial Ovarian Cancer
}

\author{
Zheng Tingting ${ }^{1,2,3,4^{*}}$, Lin Xiaojing ${ }^{1,2,3}$, Tang Xiaoyan ${ }^{1,2,3}$, Hua Keqin ${ }^{1,2,3}$ and Qiu Junjun ${ }^{1,2,3 凶}$ \\ 1. Department of Gynaecology, Obstetrics and Gynaecology Hospital, Fudan University, 419 Fangxie Road, Shanghai 200011, China Shanghai 200011, China. \\ 2. Department of Obstetrics and Gynaecology of Shanghai Medical College, Fudan University, 138 Yixueyuan Road, Shanghai 200032, China. \\ 3. Shanghai Key Laboratory of Female Reproductive Endocrine-Related Diseases, 413 Zhaozhou Road, Shanghai 200011, China. \\ 4. Department of Gynaecology, The First affiliated Hospital of Zhengzhou University, Zhengzhou University, 1 Jianshe Road, Zhengzhou 471000, Zhengzhou \\ 471000, China. \\ *First-authors.
}

$\triangle$ Corresponding authors: Jun-Jun Qiu, E-mail: qiujunjun1113@163.com; Telephone: +86018017738139; Fax: +86-021-63455090. Ke-Qin Hua, E-mail: huakeqin@fudan.edu.cn; huakeqin@126.com, Telephone: +86015921515900, Fax: +86-021-63455090.

() The author(s). This is an open access article distributed under the terms of the Creative Commons Attribution License (https://creativecommons.org/licenses/by/4.0/). See http://ivyspring.com/terms for full terms and conditions.

Received: 2019.05.13; Accepted: 2020.06.15; Published: 2020.07.09

\begin{abstract}
Antisense long noncoding RNAs serve as important regulators of protein-coding genes and contribute to tumorigenesis and metastasis. AGAP2-AS1, an antisense IncRNA transcribed from AGAP2, is involved in various cancer types. However, the clinical significance, biological roles and regulatory mechanisms of AGAP2-AS1 in epithelial ovarian cancer (EOC) have not been thoroughly elucidated to date. In this study, we demonstrated the expression pattern and biological roles of AGAP2-AS1 in EOC. Clinically, AGAP2-AS1 expression was decreased in EOC tissues compared to that in the controls. Low expression of AGAP2-ASI was associated with advanced FIGO stage, high histological grade, serous subtype and lymph node metastasis in patients with EOC. AGAP2-AS1 inhibited cell migration, invasion and proliferation in vitro. AGAP2-AS1 suppressed tumor growth in vivo. Mechanistically, AGAP2-AS1 inhibited cell metastasis and proliferation by downregulating KRAS, FGFR4, and CTSK and suppressing epithelial-mesenchymal transition. In conclusion, we provide the first evidence for the tumor-suppressing effect of AGAP2-AS1 in EOC and demonstrate that AGAP2-AS1 may represent a promising therapeutic target for EOC patients.
\end{abstract}

Key words: AGAP2-AS1; proliferation; metastasis; epithelial ovarian cancer

\section{Introduction}

Ovarian cancer $(\mathrm{OC})$ is the most lethal gynecological cancer and the fifth most common cause of cancer-related death among women [1]. Epithelial ovarian cancer (EOC) accounts for the majority of OC and is characterized by widespread and rapid metastasis in the peritoneal cavity. Despite aggressive treatment, the prognosis of EOC patients remains poor due to tumor metastasis and recurrence. Therefore, there is a demand for new insights into EOC and for research characterizing the molecular mechanisms underlying EOC progression.

Recently, long noncoding RNAs have emerged as crucial functional molecules in various cancers [2-5]. Among these lncRNAs, antisense noncoding
RNAs, a class transcribed from the antisense strand of transcriptional units, are involved in the regulation of protein-coding genes through cis or transmechanisms. Most recently, AGAP2-AS1, an antisense long noncoding RNA that has been found to serve important functions in tumorigenesis and metastasis $[6,7]$, has become the focus of our attention. AGAP2-AS1 is located on chromosome 12q14.1 and consists of 1567 nucleotides. This gene is on a locus with AGAP2 and may function as a co-regulator of AGAP2 [8-10]. It is now widely accepted that AGAP2 regulates cell migration [11], and overexpression of AGAP2 causes intracellular redistribution of activator protein 1 (AP- 1) [12], which is an important 
modulator in several carcinomas, such as breast $[13,14]$ and endometrial carcinomas [15], ovarian cancer [16], colorectal cancer [17], gastric cancer [18], acute myeloid leukemia [19] and anaplastic large cell lymphoma [20]. Despite these findings, however, the relationship between AGAP2-AS1 and AGAP2 has not been determined to date, and no published studies have investigated the clinical significance or biological function of AGAP2-AS1 in EOC.

In the present study, we examined the expression of AGAP2-AS1 in EOC tissues and ovarian cancer cell lines and analyzed the correlation between AGAP2-AS1 levels and clinicopathological features. Additionally, we investigated the functional impact of AGAP2-AS1 on tumor migration, invasion and proliferation during EOC progression through a series of in vitro and in vivo assays. Moreover, we also explored the molecular events that occurred downstream of AGAP2-AS1 involvement in EOC progression. These data provide novel insights into the role of AGAP2-AS1 in the progression of EOC and identify a potential therapeutic target in patients with EOC.

\section{Materials and Methods}

\section{Tissue samples}

Eighty EOC tissue samples were obtained from patients undergoing surgical treatment, and ten samples of normal ovarian surface epithelial (NOSE) tissue were collected as controls at the Obstetrics and Gynecology Hospital of Fudan University, China. Both written informed consent of patients and approval of the ethics committee were obtained for our study. A diagnosis of EOC was determined histologically by experienced pathologists. The 80 patients with EOC underwent standard ovarian cancer therapeutic approaches according to the guidelines, which included staging surgery and tumor cytoreductive surgery (debulking) surgery. Patients with borderline ovarian tumors or patients complicated with other gynecological tumors were excluded. No patients who had received preoperative treatment, such as radiotherapy, chemotherapy or hormonal therapy, were included in our studies. Ten NOSE tissue samples were obtained from participants diagnosed with uterine fibroids scheduled to undergo hysterectomy and oophorectomy. Patients with ovarian cysts or a history of ovarian surgery were excluded. The patients' clinicopathological data were collected by reviewing medical records.

\section{Cell lines and cell culture}

Human ovarian cancer cell lines (SKOV3-ip, OVCAR3, HO8910, HEY, ES2) were purchased from the American Type Culture Collection (Manassas, VA,
USA). All cell lines were maintained in RPMI-1640 medium (Gibco, MD, USA) containing 10\% fetal bovine serum (FBS; Gibco, CA, USA) and 100 units $/ \mathrm{ml}$ penicillin-streptomycin (Thermo Fisher Scientific, CA, USA) in a humidified $5 \% \mathrm{CO}_{2}$ incubator at $37^{\circ} \mathrm{C}$. All cell lines were passaged for fewer than 6 months in our laboratory after resuscitation.

\section{Lentiviral vector production and establishment of stable \\ AGAP2-AS1-knockdown or \\ AGAP2-AS1-overexpression tumor cells}

The recombinant AGAP2-AS1-shRNA-1/ AGAP2-AS1-shRNA-2/AGAP2-AS1-overexpression lentivirus and the negative control lentivirus were prepared and titered to $10^{9} \mathrm{TU} / \mathrm{ml}$ (transfection unit). To obtain a stable AGAP2-AS1-knockdown cell line, SKOV3.ip/OVCAR3 cells were seeded in six-well dishes at a density of $3 \times 10^{3}$ cells per well. The cells were then infected with virus at the same titer with $5 \mu \mathrm{g} / \mathrm{ml}$ polybrene (GenePharma, shanghai, China) on the following day. Approximately $72 \mathrm{~h}$ after viral transfection, the culture medium was replaced with selection medium containing $2 \mu \mathrm{g} / \mathrm{ml}$ puromycin. The cells were then cultured for at least 14 days. The puromycin-resistant cell clones were isolated, amplified in medium containing $1 \mu \mathrm{g} / \mathrm{ml}$ puromycin for seven to nine days and transferred to a medium without puromycin. The clones were designated LV (AGAP2-AS1- knockdown) cells or NC (negative control) cells. The target sequences for AGAP2-AS1 shRNAs were 5'- GCTCTGTTCCCTCACGCTTAC (AGAP2-AS1-shRNA-1) and 5'- GGCATTCACTA CACTCCCTAG -3' (AGAP2-AS1-shRNA-2). The efficiency of AGAP2-AS1 knockdown was confirmed via quantitative real-time polymerase chain reaction (qRT-PCR).

\section{RNA isolation, reverse transcription, and qRT-PCR}

Total RNA was extracted using TRIzol reagent (Invitrogen, CA, USA). RNA was reverse transcribed into cDNA using a Prime-ScriptTM one step RT-PCR kit (TAKARA, Dalian, China). AGAP2-AS1 expression levels were measured with $\mathrm{qPCR}$ in quadruplicate using SYBR Green PCR Master Mix (TAKARA, Dalian, China) on an ABI StepOne RealTime PCR machine (Applied Biosystems, CA, USA). GAPDH was used as an endogenous standard. The sequences of the primers designed by Shanghai Engineering Biotech Corp (Shanghai, China) are listed as follows: AGAP2-AS1 forward primer, 5'TACCTTGACCTTGCTGCTCTC- $3^{\prime}$ and reverse primer, 5'- TGTCCCTTAATGACCCCATCC-3'; and GAPDH forward primer, 5'- GGGAAGGTGAAGG 
TCGGAGT-3' and reverse primer, 5' - GGGGTCATTG ATGGCAACA-3'.

\section{Cell migration and invasion assays}

Migration and invasion of EOC cells were evaluated in vitro using a modified Boyden chamber Transwell system (Corning, MA, USA). Twenty-four hours after transfection, cells were seeded in Transwell plates for further experiments. For migration, the upper chamber was plated with $5 \times 10^{4}$ cells in $200 \mu \mathrm{L}$ serum-free RPMI 1640, and the lower chamber was filled with $500 \mu \mathrm{L}$ RPMI 1640 with $10 \%$ fetal bovine serum. After $24 \mathrm{~h}$ of incubation at $37^{\circ} \mathrm{C}$, the cells on the upper surface of the filter were removed using cotton swabs. The migrated cells that remained on the bottom surface were fixed with methanol for 30 minutes and then stained with $0.1 \%$ crystal violet for 10 minutes. The number of cells in five random fields of each chamber was counted and imaged using a digital microscope (magnification, $x$ 200). The average number of cells was calculated.

For the Matrigel invasion assay, the infected cells $\left(1 \times 10^{5}\right)$ were plated in the top chamber of Transwell assay inserts (Corning, MA, USA) with a Matrigelcoated membrane containing $8 \mu \mathrm{m}$ pores in $200 \mu \mathrm{L}$ of serum-free RPMI 1640 medium. The assays were conducted in triplicate.

\section{Cell proliferation assays}

The effect of AGAP2-AS1 on cell proliferation was determined using real-time cellular analysis (RTCA). Twenty-four hours after transfection, cells (5 $\times 10^{3}$ cells/well) were seeded into several E-Plate 16 (ACEA Biosciences Inc., USA) for proliferation assays according to the manufacturer's recommendations. The plates were kept in a cell incubator at $37^{\circ} \mathrm{C}$ and $5 \% \mathrm{CO}_{2}$ for $4-6$ days. The cell index and growth curves were automatically recorded on an xCELLigence RTCA System (Roche, USA).

\section{Microarray analysis}

Seventy-two hours after transfection of AGAP2AS1 shRNA2 lentivirus or NC lentivirus, total RNA from SKOV3.ip and OVCAR3 cells was extracted using TRIzol reagent (Invitrogen, CA, USA). Then, the $\mathrm{RT}^{2}$ ProfilerTM PCR Array Human Tumor Metastasis Array (PAHS-028Z) was used to identify the differentially expressed genes of AGAP2-AS1 knockdown (KD) and control samples (NC). Differentially expressed genes, Gene Ontology (GO) and KEGG pathway analyses were used to investigate the functional significance of the molecular changes.

\section{Western blot assays}

To analyze the expression of genes downstream of AGAP2-AS1, Western blot analyses were performed using the following primary antibodies: rabbit anti-human KRAS (Abcam; 1:200); anti-FGFR4 (Abcam; 1:1000); anti-CTSK (Abcam; 1:1000); anti-E-cadherin (Abcam; 1:50); anti-vimentin (Abcam; 1:2000) and anti-GAPDH (Abcam; 1:10000). Briefly, stimulated cells were lysed with RIPA buffer containing protease inhibitors (Roche, CA, USA); 20$30 \mu \mathrm{g}$ samples of the lysates were separated on $10 \%$ SDS-PAGE gels and transferred to PVDF membranes. The membranes were incubated with primary antibodies overnight at $4{ }^{\circ} \mathrm{C}$. The primary antibody incubation was followed by incubation with an HRP-conjugated secondary antibody. The bound antibodies were detected using an ECL kit (Tangon, shanghai, China).

\section{In vivo experiment}

All animal studies were approved by the Institutional Animal Care and Use Committee of the Fudan University. Twelve female athymic Balb/c nude mice (aged 4 to 6 weeks, weighing 14-18 g) were purchased from Department of Laboratory Animal, Fudan University, housed in a pathogen-free animal facility and randomly assigned to the negative control lentivirus (SKOV3.ip-NC) group or the AGAP2-AS1knockdown (SKOV3.ip-KD) group (six mice per group). A total of $10^{7}$ SKOV3.ip cells were subcutaneously injected into each mouse. The engrafted mice were inspected weekly for signs of distress or changes in weight and euthanized four weeks after the subcutaneous injection of the tumor cells.

\section{Statistical analysis}

Student's t-test (two-tailed) was performed to analyze the in vitro data using GraphPad software. The relationship between AGAP2-AS1 expression levels and clinical parameters was assessed with the Pearson's correlation coefficients. All data are presented as the mean $\pm \mathrm{SD}$. $P$-values less than 0.05 were considered to be statistically significant.

\section{Results}

\section{AGAP2-AS1 expression decreases in epithelial ovarian cancer tissues and correlates with EOC progression}

To identify the role of AGAP2-AS1 in EOC, we first analyzed the GSE9891 microarray data obtained from the GEO database and found that AGAP2-AS1 was significantly downregulated in the 267 ovarian cancer tissues compared to the 18 ovarian borderline tumors (Fig. 1A-1B). Furthermore, we detected the expression of AGPA2-AS1 in 80 EOC tissues. As shown in Fig. 1C, AGAP2-AS1 expression decreased in most $(86.25 \%, 69 / 80)$ EOC tissues compared to that 
in the normal ovarian samples, indicating that AGAP2-AS1 might play a part in EOC progression. To explore the correlation between AGAP2-AS1 levels and EOC malignant features, 80 EOC patients were divided into the AGAP2-AS1 high group $(\mathrm{n}=40)$ and the AGAP2-AS1 low group $(\mathrm{n}=40)$ using the median value (Fig. 1D). We found that low AGAP2-AS1 levels were associated with advanced FIGO stage, high histological grade, lymph node metastasis and serous subtype (Table 1), indicating that AGAP2-AS1 negatively correlates with a high degree of malignancy of EOC. Collectively, these results suggested that AGAP2-AS1 might act as a tumor suppressor in EOC.

Table 1. Association of AGAP2-AS1 expression with clinicopathological variables in EOC patients

\begin{tabular}{|c|c|c|c|}
\hline \multirow[t]{3}{*}{ Variable } & Low AGAP2-AS1 & High AGAP2-AS1 & $P$ \\
\hline & Expression, $\mathrm{n}(\%)$ & Expression, $\mathrm{n}(\%)$ & \\
\hline & $\mathrm{n}=40$ & $\mathrm{n}=40$ & \\
\hline \multicolumn{4}{|l|}{ Age (years) } \\
\hline$<50$ & 13 & 12 & 0.809 \\
\hline$\geq 50$ & 27 & 28 & \\
\hline \multicolumn{4}{|l|}{ Histological subtype } \\
\hline Serous & 39 & 28 & 0.001 \\
\hline Other & 1 & 12 & \\
\hline \multicolumn{4}{|l|}{ FIGO stage } \\
\hline I-II & 7 & 15 & 0.045 \\
\hline III-IV & 33 & 25 & \\
\hline \multicolumn{4}{|l|}{ Histological grade } \\
\hline High & 39 & 31 & 0.007 \\
\hline Low & 1 & 9 & \\
\hline Residual tumor diameter (cm) & & & 0.396 \\
\hline$<1$ & 36 & 38 & \\
\hline$\geq 1$ & 4 & 2 & \\
\hline \multicolumn{4}{|l|}{ Lymph node metastasis } \\
\hline Absent & 20 & 29 & 0.039 \\
\hline Present & 20 & 11 & \\
\hline \multicolumn{4}{|l|}{ CA125 level (U/ml) } \\
\hline$<600$ & 18 & 20 & 0.654 \\
\hline$\geq 600$ & 22 & 20 & \\
\hline \multicolumn{4}{|l|}{ Ascites } \\
\hline$<100$ & 21 & 24 & 0.499 \\
\hline$\geq 100$ & 19 & 16 & \\
\hline
\end{tabular}

NOTE: The median value of AGAP2-AS1 expression was used as a cut-off for the low/high AGAP2-AS1 expression groups.

\section{AGAP2-AS1 inhibits EOC cell migration, invasion and proliferation}

Based on the clinical observations, we hypothesized that downregulation of APAP2-AS1 promoted EOC progression. To this end, we measured the expression patterns of AGAP2-AS1 in EOC cell lines. The qRT-PCR results showed that AGAP2-AS1 was downregulated in the EOC cell lines (SKOV3.ip, OVCAR3, HO8910, HEY, ES2) compared with the normal human ovarian epithelial cell line (HOSEpiC) (Fig. 2A). Furthermore, the expression levels of AGAP2-AS1 in three pairs of cell lines (HO8910 vs HO8910-PM, SKOV3 vs SKOV3.ip, HEY vs HEY-A8) were detected, and AGAP2-AS1 expression was found to be decreased in highly invasive EOC cell lines (HO8910-PM, SKOV3.ip, HEY-A8) compared to their corresponding controls (HO8910, SKOV3, HEY) (Fig. 2B). These findings collectively suggested that AGAP2-AS1 might be involved in the aggressive biological behaviors of EOC cells.

To investigate whether AGAP2-AS1 plays a role in cell migration, invasion and proliferation in EOC cells, AGAP2-AS1 was knocked down or overexpressed by lentivirus transfection. The results showed that the lentiviruses exerted a satisfactory silencing/overexpressing effect (Fig. 2C). LV2 showed a superior knockdown efficiency and was used for further tests. The migration assay showed that downregulation of AGAP2-AS1 promoted the migration of SKOV3.ip and OVCAR3 cells, while upregulation of AGPA2-AS1 suppressed HO8910 migration (Fig. 3A). The Matrigel invasion assay indicated that the suppression of AGAP2-AS1 expression significantly increased the invasive ability of SKOV3.ip and OVCAR3 cells. Conversely, overexpression of AGAP2-AS1 decreased the invasiveness of HO8910 cells (Fig. 3B). We therefore concluded that silencing AGAP2-AS1 promotes EOC cell migration and invasion in vitro. Additionally, we performed RTCA to determine whether AGAP2-AS1 is involved in cell proliferation. As expected, cell proliferation assays showed that AGAP2-AS1 silencing significantly promoted the proliferation of SKOV3.ip and OVCAR3 cells (Fig. 4). However, overexpressed AGAP2-AS1 has a slight suppressive effect on HO8910 cell growth. In summary, these results suggest that AGAP2-AS1 inhibits cell migration, invasion and proliferation in vitro.

\section{AGAP2-AS1 suppresses EOC growth in vivo}

To assess the effect of AGAP2-AS1 in vivo, a nude mouse xenograft model was established. SKOV3.ip cells with (KD) or without (NC) AGAP2-AS1 knockdown were injected subcutaneously into mice. The results showed that AGAP2-AS1 was effectively downregulated in SKOV3.ip-KD cells compared with that in SKOV3.ip-NC cells (Fig. 5A and B), and the silencing efficiency was confirmed after tumor removal (Fig. 5E). The downregulation of AGAP2AS1 increased the volume of tumors (Fig. 5C). The tumor weight in the KD group was significantly elevated compared with the NC group (Fig. 5D). To summarize, these results indicate that silencing AGAP2-AS1 promoted tumor growth in vivo.

\section{AGAP2-AS1 suppresses EMT and dysregulates KRAS, CTSK and FGFR4 in EOC progression}

To further illuminate the possible mechanisms through which AGAP2-AS1 contributed to EOC 
progression, gene expression profiles of OVCAR3-KD cells were compared with those of OVCAR3-NC cells using real-time PCR array analysis (PAHS-028Z). The data were further validated by qRT-PCR, and the results showed that KRAS, CTSK, and FGFR4 were markedly dysregulated (>2-fold) after AGAP2-AS1 silencing in both OVCAR3 and SKOV3.ip cells (Fig. 6A-B). Additionally, consistent with the array results, increased KRAS, CTSK, and FGFR4 protein levels were detected in both SKOV3.ip and OVCAR3 cells after AGAP2-AS1 knockdown (Fig. 6C). Furthermore, E-cadherin was downregulated and vimentin was up-regulated after AGAP2-AS1 knockdown (Fig. 6C), suggesting that AGAP2-AS1 suppressed epithelialmesenchymal transition. Taken together, these data indicate that AGAP2-AS1 regulates EOC cell migration and invasion by suppressing EMT and decreasing cell proliferation by regulating KRAS, CTSK and FGFR4.

\section{Discussion}

LncRNA, as a major component of noncoding RNAs, has attracted considerable attention in recent years. AGAP2-AS1 is an antisense RNA that co-regulates the function of AGAP2 [8-10]. It is now widely accepted that AGAP2 regulates focal adhesion kinase (FAK) activity and focal adhesion disassembly during cell migration [11]. In addition, overexpression of AGAP2 induced AP1 and was associated with metastasis and proliferation in various tumors [20]. Recently, AGAP2-AS1 was reported to be involved in tumorigenesis and metastasis [6,7]. However, no studies have reported the presence of AGAP2-AS1 in ovarian cancer, and no published studies have investigated the clinical significance or biological function of AGAP2-AS1 in EOC.

In our study, we found that AGAP2-AS1 expression significantly decreased in EOC tissues compared with that in noncancerous tissues. With a thorough analysis of clinical data, we found that low expression levels of AGAP2-AS1 are associated with advanced FIGO stage, high histological grade, lymph node metastasis and serous histological subtype. These findings indicate that lower expression of AGAP2-AS1 may promote aggressiveness and metastasis of EOC and may serve as a tumor suppressor in patients with EOC.
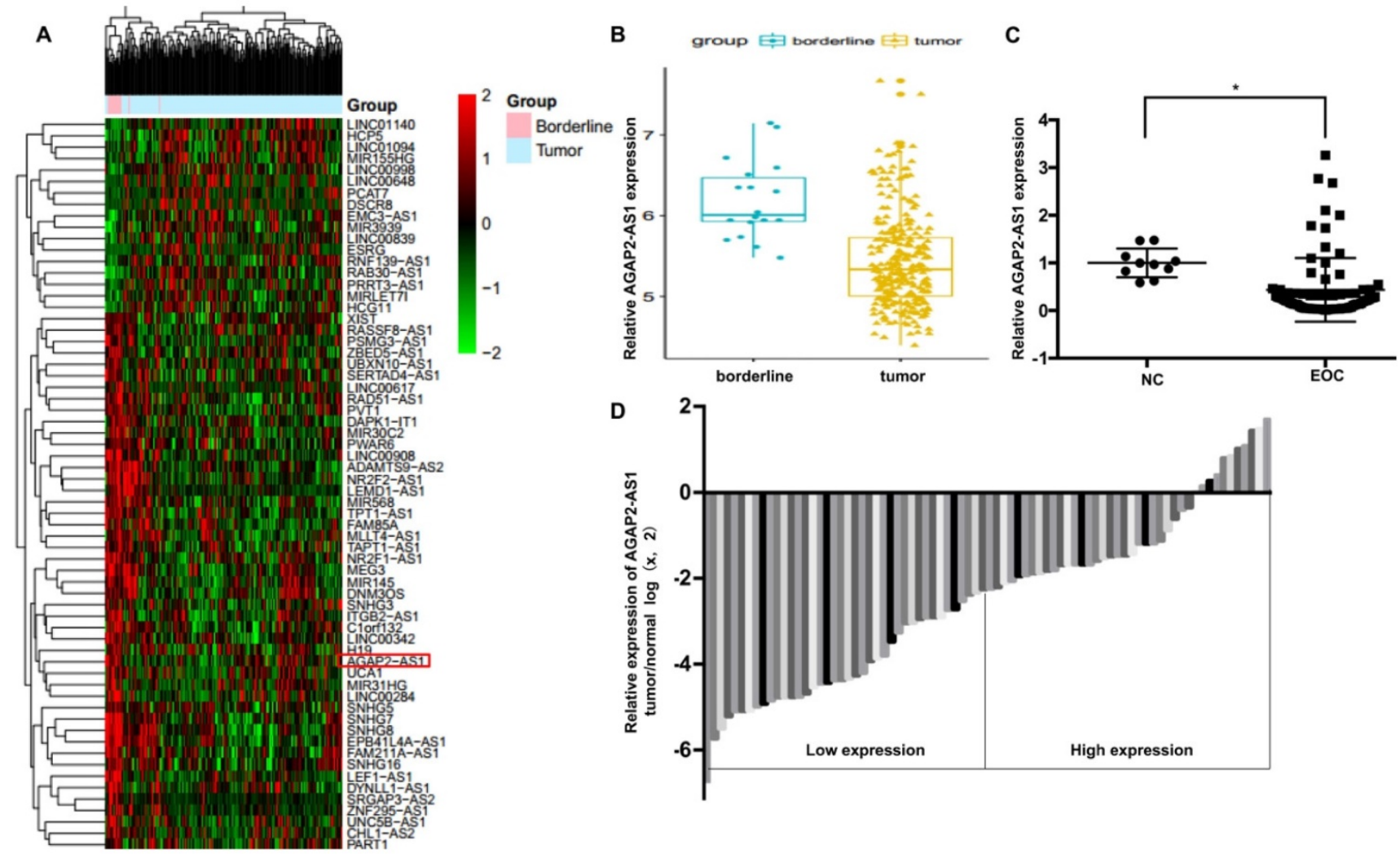

Figure 1. AGAP2-AS1 was downregulated in EOC tissues. (A) Differentially expressed IncRNAs between ovarian cancer (OC) tissues ( $\mathrm{n}=267)$ and ovarian borderline tumors $(n=18)$. (B) AGAP2-AS1 decreased in OC tissues compared to ovarian borderline tumors $(P<0.05$, Student's t-test). (C) AGAP2-AS1 was downregulated in epithelial ovarian cancer samples $(n=80)$ compared to that in normal ovarian epithelial tissues $(n=10)(P<0.05$, Student's t-test). (D) EOC patients were divided into an AGAP2-AS1 high group $(n=40)$ and an AGAP2-AS1 low group $(n=40)$ using the median value of the AGAP2-AS1 expression levels. 


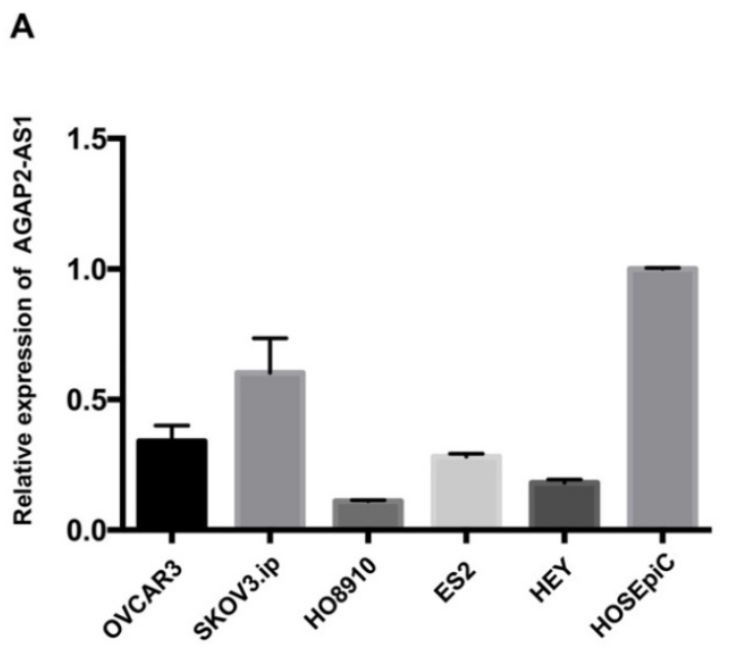

\section{B}
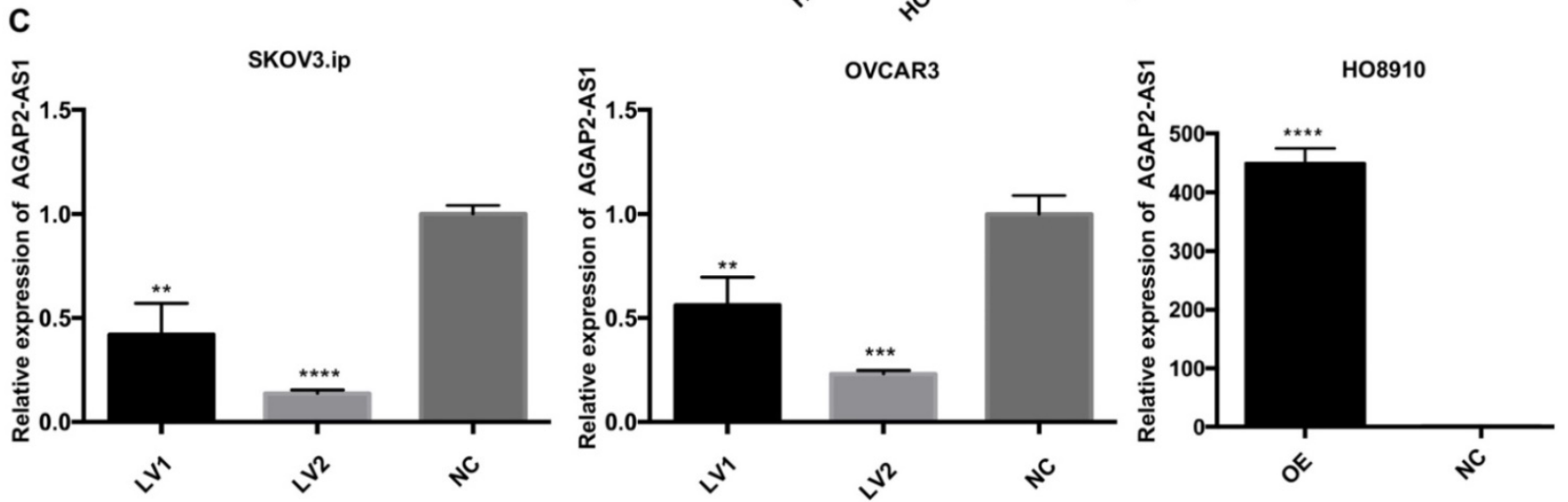

Figure 2. Expression of AGAP2-AS1 in EOC cell lines. (A) Relative AGAP2-AS1 expression in EOC cell lines. (B) AGAP2-AS1 was suppressed in highly invasive cell lines (HO8910-PM, SKOV3.ip, HEY-A8) compared to that in the controls (HO8910, SKOV3, HEY). (C) AGAP2-AS1 was significantly silenced/overexpressed by specific lentiviruses in SKOV3.ip, OVCAR3 and HO8910 cells and verified by qRT-PCR verification.

Previous studies showed that upregulation of AGAP2-AS1 promoted cell proliferation and metastasis in pancreatic cancer, glioma, gastric cancer and non-small-cell lung cancer $[6,7,21,22]$. Overexpression of AGAP2-AS1 could cause trastuzumab resistance in breast cancer [23]. However, in our study, the results showed that AGAP2-AS1 is downregulated in highly invasive EOC cell lines compared to the controls. Knockdown of AGAP2-AS1 promoted the migration, invasion and proliferation of SKOV3.ip and OVCAR3. We found that mice injected with AGAP2-AS1-KD cells developed significantly larger tumor implants compared to the controls. Silencing of AGAP2-AS1 led to a dramatic decrease in tumor weight. These results collectively demonstrated that AGAP2-AS1 is a potential tumor suppressor in EOC and that knockdown of AGAP2-AS1 contributes to EOC proliferation and metastasis. Our results are in contrast to the previously reported roles of AGAP2-AS2 in other cancers and indicate the complicated roles of lncRNAs in cancers.
As is well-known, long antisense noncoding RNAs were found to serve as regulators of their sense counterparts. For example, antisense noncoding RNA p15AS induced p15 silencing and promoted cell growth in leukemia [24]. In breast cancer, RAD51-AS1 exhibited an opposite effect in regulating the expression of RAD51 and induced RAD51-dependent double-strand break repair in malignancy [25]. Consistent with these findings, we found that the expression of AGAP2-AS1 in EOC tissues is negatively correlated with that of AGAP2. AGAP2 is known to be a proto-oncogene and is involved in the activity of AKT, NF-kB, p53, AMPK, and FAK [11,26-29]. Furthermore, researchers found that AGAP2 promoted cell proliferation, migration, and invasion and suppressed cell apoptosis $[30,31]$. As the antisense lncRNA of AGAP2, AGAP2-AS1 was found to conversely inhibited cell proliferation and metastasis in EOC cell lines, and low expression of AGAP2-AS1 was correlated with tumor malignant features, indicating that AGAP2-AS1 is a potential tumor suppressor of EOC. 
A

ठั

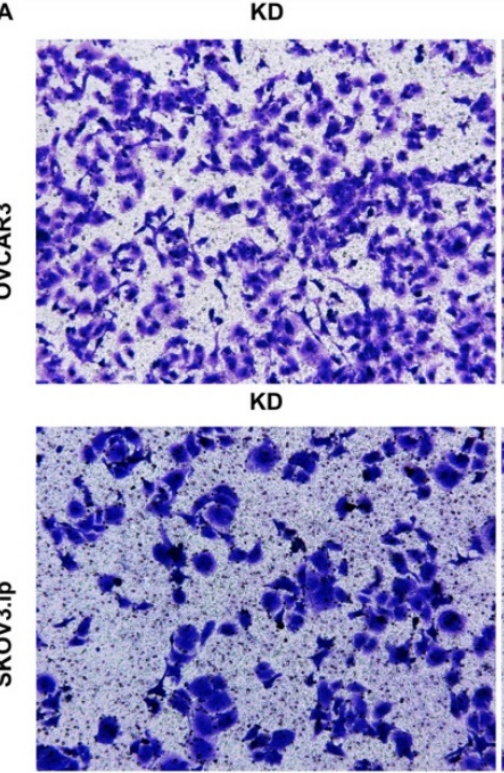

$\mathrm{OE}$

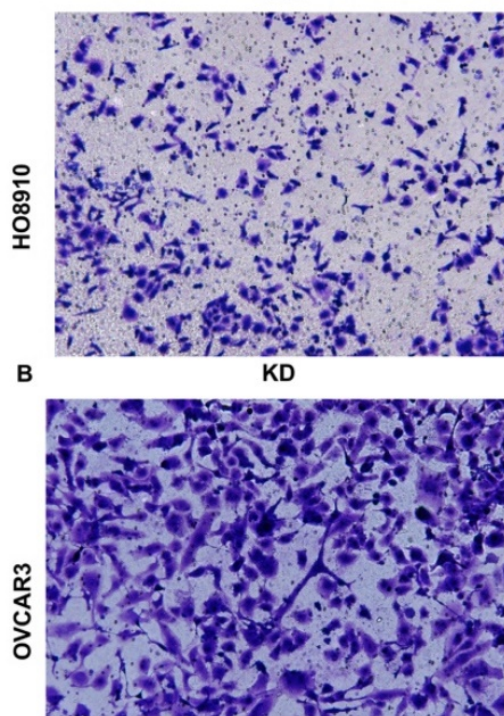

KD

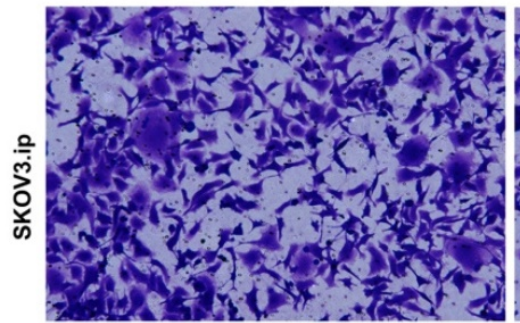

OE

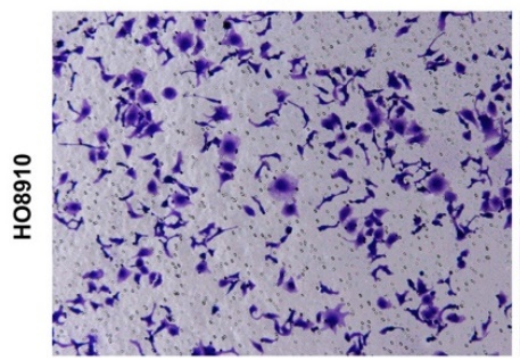

NC

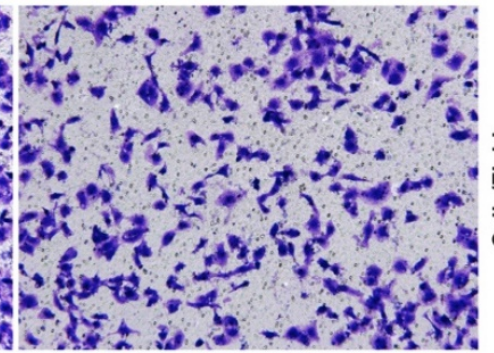

NC

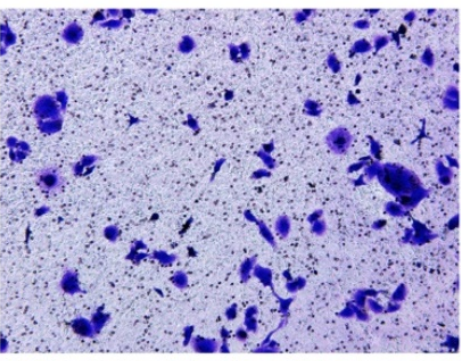

NC

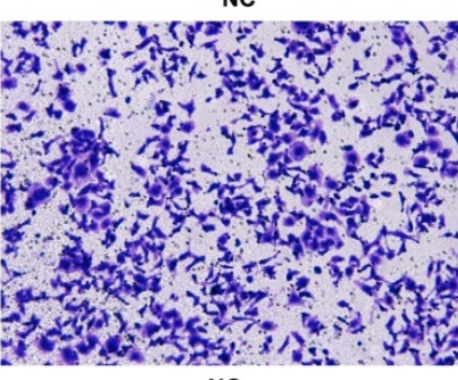

NC

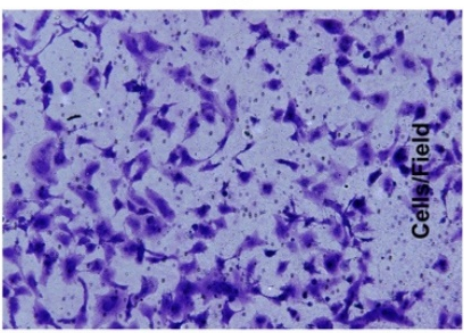

NC

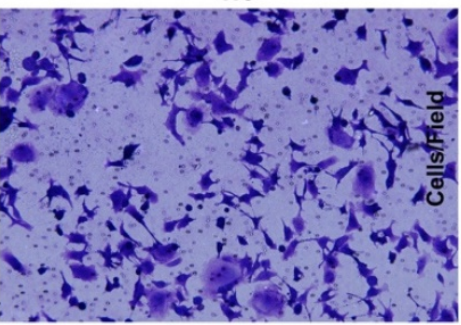

NC

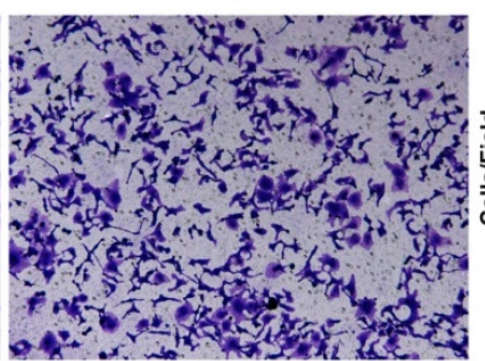

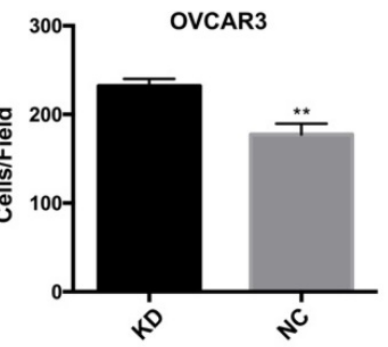
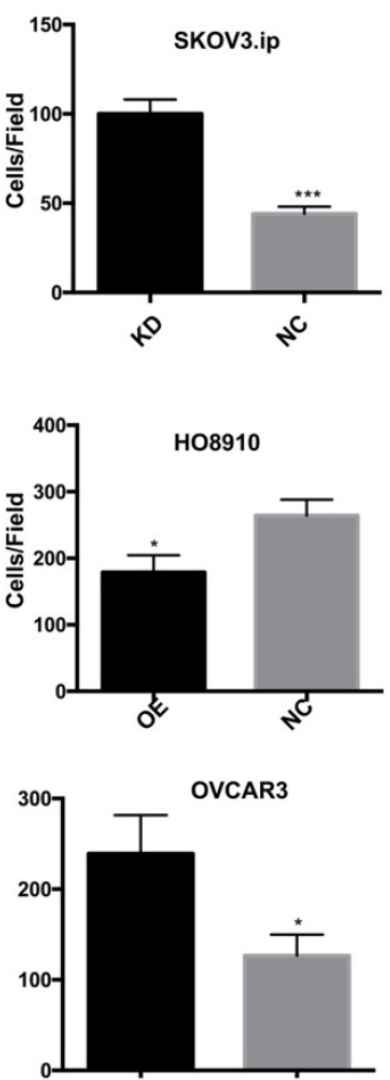

요

से
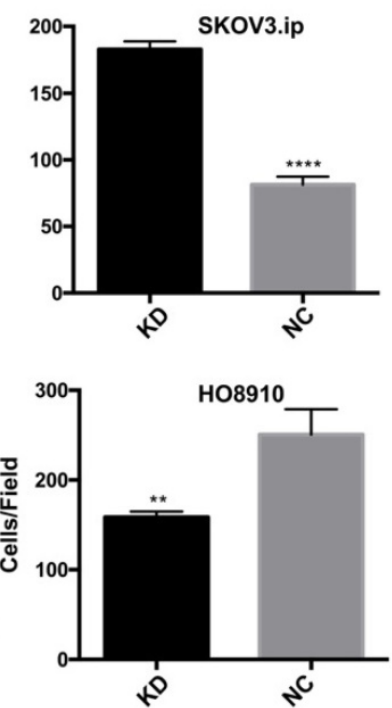

Figure 3. AGAP2-AS1 regulated cell migration, invasion in SKOV3.ip, OVCAR3 and HO8910 cells. (A) AGAP2-AS1 silencing promoted cell migration of SKOV3.ip and OVCAR3 cells, and AGAP2-AS1 overexpression inhibited cell migration of HO8910 cells. (B) A Matrigel invasion assay showed that knockdown of AGAP2-AS1 increased the invasive abilities of SKOV3.ip and OVCAR3 cells and that upregulation of AGAP2-AS1 inhibited the invasion of HO8910 cells. 

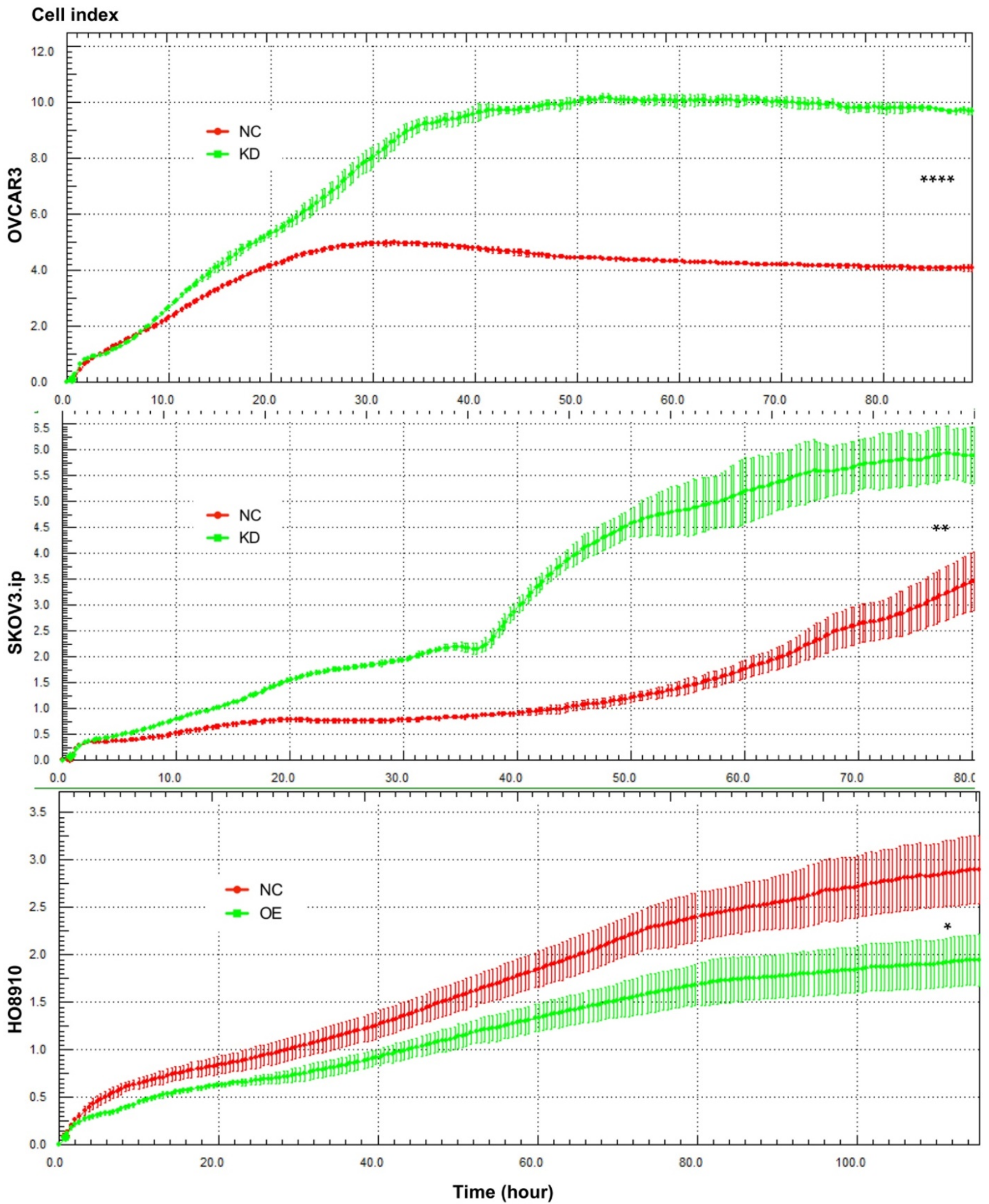

Figure 4. AGAP2-AS1 regulated cell proliferation in SKOV3.ip, OVCAR3 and HO8910 cells. Proliferation assays showed that downregulation of AGAP2-AS1 contributed to cell proliferation in SKOV3.ip and OVCAR3 cells, while upregulation of AGAP2-AS1 suppressed HO8910 cell growth. Each experiment was repeated in triplicate $(n=3)$. (The results are shown as the mean \pm SD; $* P<0.05 ; * * P<0.01 ; * * * P<0.001 ; * * * * P<0.0001$; Student's t-test).

The contradictory roles of lncRNAs in different cancers open up new possibilities for achieving a deep understanding of their functions and research methods. For example, MALAT1, which has previously been described as a metastasis-promoting lncRNA, has recently been found to suppress breast cancer metastasis [32]. The opposite functions of lncRNA in different cancers may be attributed to the tissue-specific expression pattern and regulatory diversities. In addition, different knockdown techniques may lead to different results. 


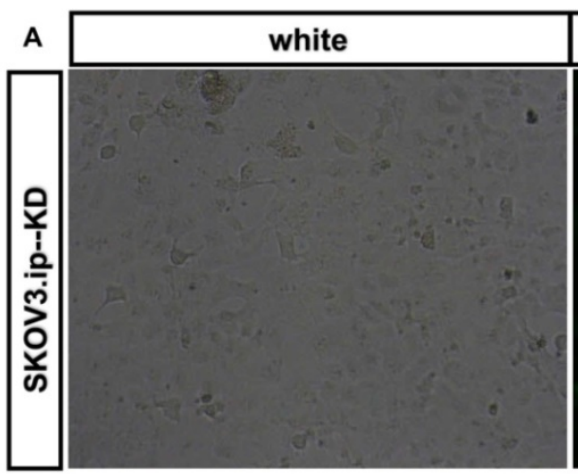

C

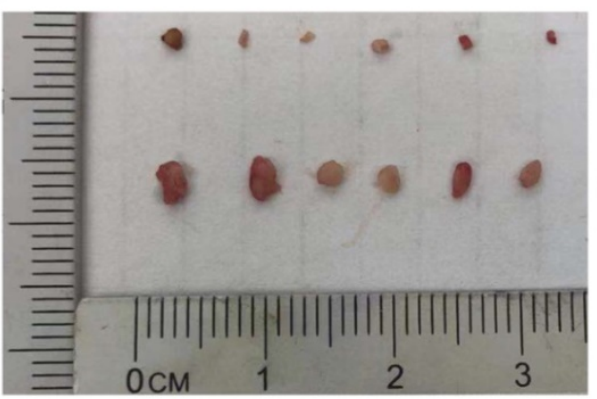

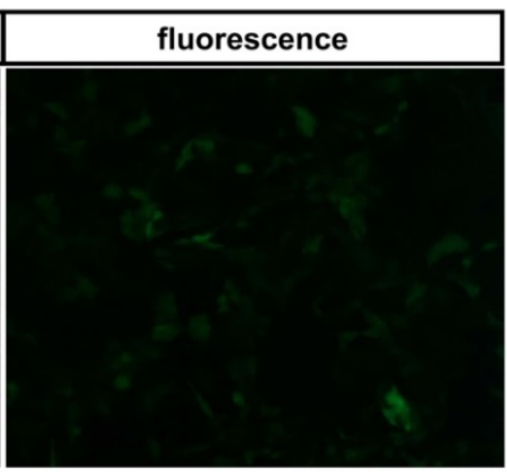

D

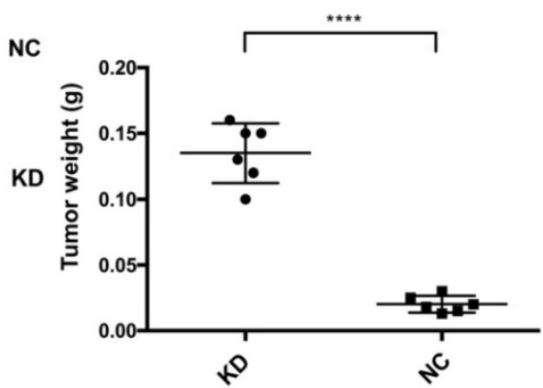

B

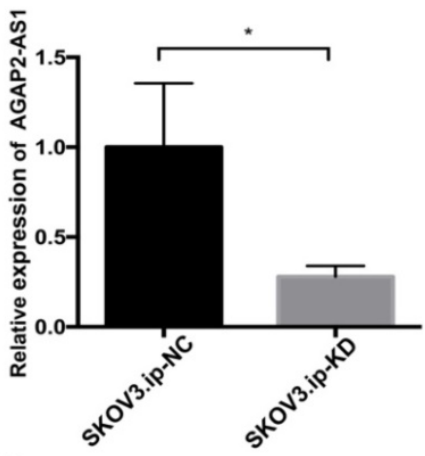

E

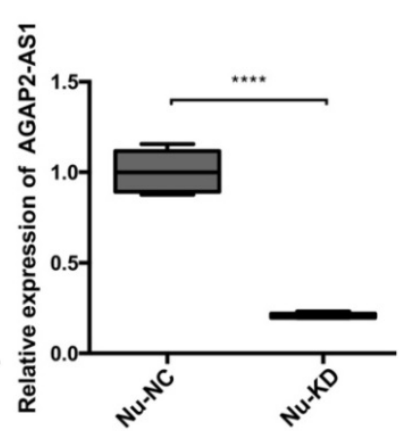

Figure 5. AGAP2-AS1 knockdown promoted tumor growth in vivo. (A) The efficiency of lentivirus transfection was visually assessed by microscopy. (B) The knockdown efficiency of SKOV3.ip-KD was normalized to SKOV3.ip-NC with qRT-PCR confirmation. (C) Tumor volumes in the LV-KD group were significantly larger than those in the LV-NC group. (D) Tumor weight in the LV-KD group was significantly increased compared with that in the LV-NC group. (E) The relative expression of AGAP2-AS1 in the Nu-KD group was significantly decreased compared with that in the Nu-NC group. (LV-KD: AGAP2-AS1-knockdown lentivirus cells, LV-NC: AGAP2-AS1-negative control lentivirus cells $* P<0.05 ; * * * P<0.0001$ by Student's t-test)

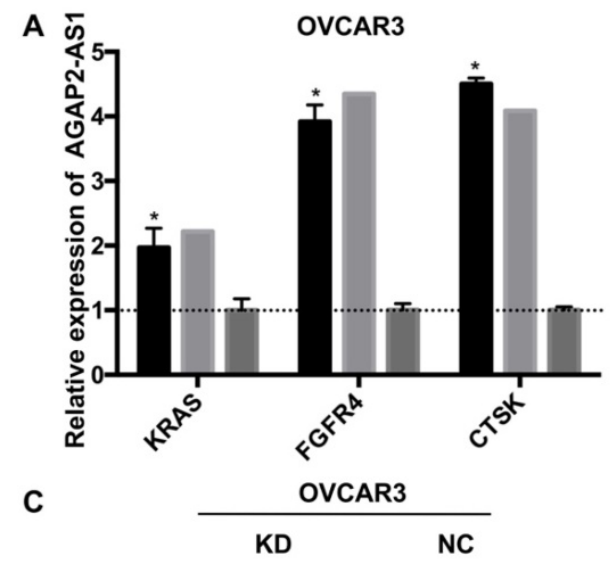

CTSK
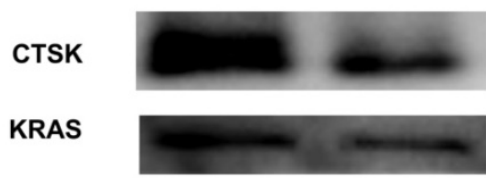

FGFR4

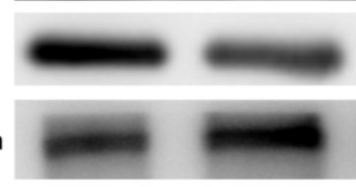

Vimentin

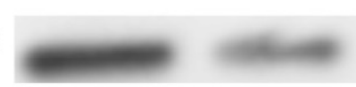

GAPDH

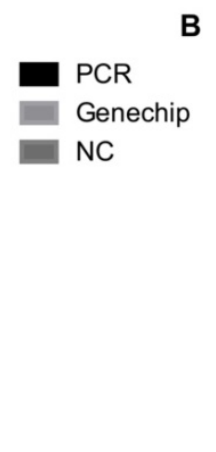

SKOV3.ip

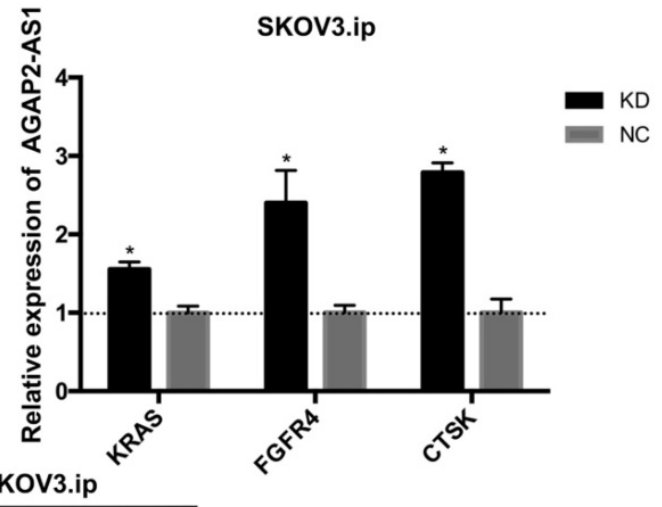

KD

NC

CTSK

KRAS

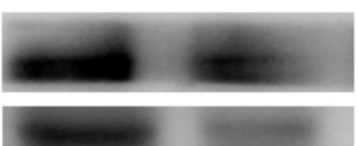

FGFR4

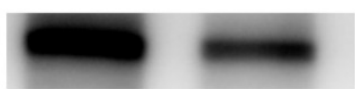

E-cadherin

Vimentin
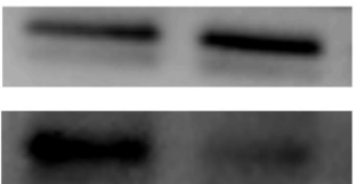

GAPDH

Figure 6. AGAP2-AS1 knockdown regulated KRAS, CTSK, and FGFR4 expression in SKOV3.ip and OVCAR3 cells and induced epithelial-mesenchymal transition. (A) KRAS, FGFR4, and CTSK were significantly upregulated in SKOV3.ip and OVCAR3 cells after AGAP2-AS1 silencing, which was consistent with the PCR array results. (B) The protein levels of KRAS, FGFR4, and CTSK were detected by Western blotting after AGAP2-AS1 knockdown. After AGAP2-AS1 knockdown, E-cadherin levels were reduced, and vimentin levels were increased. All results are shown as the mean \pm SD and are representative of three independent experiments. $(* P<0.05$, Student's $t$-test). 
To date, our understanding of the molecular mechanisms of lncRNAs in tumor progression is far from enough. Previous studies have demonstrated that lncRNAs promote cancer metastasis and proliferation by regulating protein-coding genes. As expected, our results demonstrated that the expression of EMT-related genes (FGFR4, CTSK, E-cadherin, Vimentin) and oncogenic genes (KRAS) were dysregulated by AGAP2-AS1 knockdown in EOC cell lines (SKOV3.ip and OVCAR3), implying that these genes participate in AGAP2-AS1-induced EOC progression.

However, further research is needed to elucidate the regulatory mechanisms underlying the relationship between AGAP2-AS1 and its target genes. In addition, studies with larger samples are required to explore the prognostic value of AGAP2AS1 in EOC patients.

In conclusion, our data provide evidence for the involvement of AGAP2-AS1 in EOC proliferation and metastasis and suggest that AGAP2-AS1 may be a tumor suppressor in EOC. AGAP2-AS1-based therapeutic strategies aimed at upregulating suppressive lncRNAs may represent a promising alternative therapeutic approach for the treatment of patients with EOC.

\section{Abbreviations}

AP-1: activator protein 1; CTSK: cathepsin K; EMT: epithelial-mesenchymal transition; EOC: epithelial ovarian cancer; FGFR4: fibroblast growth factor receptor 4; FBS: fetal bovine serum; FIGO: International Federation of Gynecology and Obstetrics; GO: Gene Ontology; KEGG: Kyoto Encyclopedia of Genes and Genomes; lncRNA: long noncoding RNA; NOSE: normal ovarian surface epithelial tissues; OC: ovarian cancer; RTCA: realtime cellular analysis.

\section{Acknowledgements}

This project was supported by funding from the National Natural Science Foundation of China (No. 81971361; to Jun-jun Qiu), the Natural Science Foundation of Shanghai Science and Technology (No. 19ZR1406900; to Jun-jun Qiu), and shanghai "Rising Stars of Medical Talent" Youth Development Program to Jun-jun Qiu, the Research and Innovation Project of Shanghai Municipal Education Commission (No. 2019-01-07-00-07-E00050; to Ke-qin Hua), and the artificial Intelligence Innovation Project of Shanghai Municipal Commission of Economy and Informatization (No. 2018-RGZN-02041; to Ke-qin Hua).

\section{Competing Interests}

The authors have declared that no competing interest exists.

\section{References}

1. Siegel RL, Miller KD, Jemal A. Cancer statistics, 2017. CA Cancer J Clin. 2017; 67: 7-30.

2. Yang ZG, Gao L, Guo XB, Shi YL. Roles of long non-coding RNAs in gastric cancer metastasis. World J Gastroenterol. 2015; 21: 5220-30.

3. Li G, Zhang H, Wan X, Yang X, Zhu C, Wang A, et al. Long noncoding RNA plays a key role in metastasis and prognosis of hepatocellular carcinoma. Biomed Res Int. 2014; 2014: 780521.

4. Wu T, Yin X, Zhou Y, Wang Z, Shen S, Qiu Y, et al. Roles of noncoding RNAs in metastasis of nonsmall cell lung cancer: a mini review. J Cancer Res Ther. 2015; 11 Suppl 1: C7-10.

5. Li H, Ma SQ, Huang J, Chen XP, Zhou HH. Roles of long noncoding RNAs in colorectal cancer metastasis. Oncotarget. 2017; 8: 39859-76.

6. Li W, Sun M, Zang C, Ma P, He J, Zhang M, et al. Upregulated long non-coding RNA AGAP2-AS1 represses LATS2 and KLF2 expression through interacting with EZH2 and LSD1 in non-small-cell lung cancer cells. Cell Death Dis. 2016; 7: e2225.

7. Qi F, Liu X, Wu H, Yu X, Wei C, Huang X, et al. Long noncoding AGAP2-AS1 is activated by SP1 and promotes cell proliferation and invasion in gastric cancer. J Hematol Oncol. 2017; 10: 48.

8. Chan J, Atianand M, Jiang Z, Carpenter S, Aiello D, Elling R, et al. Cutting edge: a natural antisense transcript, AS-IL1alpha, controls inducible transcription of the proinflammatory cytokine IL-1alpha. J Immunol. 2015; 195: 1359-63.

9. Kimura T, Jiang S, Nishizawa M, Yoshigai E, Hashimoto I, Nishikawa M, et al. Stabilization of human interferon-alpha1 mRNA by its antisense RNA. Cell Mol Life Sci. 2013; 70: 1451-67.

10. Peng H, Liu Y, Tian J, Ma J, Tang X, Rui K, et al. The long noncoding RNA IFNG-AS1 promotes $\mathrm{T}$ helper type 1 cells response in patients with Hashimoto's thyroiditis. Sci Rep. 2015; 5: 17702.

11. Zhu Y, Wu Y, Kim JI, Wang Z, Daaka Y, Nie Z. Arf GTPase-activating protein AGAP2 regulates focal adhesion kinase activity and focal adhesion remodeling. J Biol Chem. 2009; 284: 13489-96.

12. Nie Z, Fei J, Premont RT, Randazzo PA. The Arf GAPs AGAP1 and AGAP2 distinguish between the adaptor protein complexes AP-1 and AP-3. J Cell Sci. 2005; 118: 3555-66.

13. Bland KI, Konstadoulakis MM, Vezeridis MP, Wanebo HJ. Oncogene protein co-expression. Value of Ha-ras, c-myc, c-fos, and p53 as prognostic discriminants for breast carcinoma. Ann Surg. 1995; 221: 706-18; discussion 18-20.

14. Kharman-Biz A, Gao H, Ghiasvand R, Zhao C, Zendehdel K, Dahlman-Wright $\mathrm{K}$. Expression of activator protein-1 (AP-1) family members in breast cancer. BMC Cancer. 2013; 13: 441.

15. Bamberger AM, Milde-Langosch K, Rossing E, Goemann C, Loning T. Expression pattern of the AP-1 family in endometrial cancer: correlations with cell cycle regulators. J Cancer Res Clin Oncol. 2001; 127: 545-50.

16. Oliveira-Ferrer L, Rossler K, Haustein V, Schroder C, Wicklein D, Maltseva D, et al. c-FOS suppresses ovarian cancer progression by changing adhesion. Br J Cancer. 2014; 110: 753-63.

17. Schewe DM, Biller T, Maurer G, Asangani IA, Leupold JH, Lengyel ER, et al. Combination analysis of activator protein-1 family members, Sp1 and an activator protein-2alpha-related factor binding to different regions of the urokinase receptor gene in resected colorectal cancers. Clin Cancer Res. 2005; 11: 8538-48.

18. Zhou L, Zhang JS, Yu JC, Cui QC, Zhou WX, Kang WM, et al. Negative association of c-FOS expression as a favorable prognostic indicator in gastric cancer. Arch Med Res. 2010; 41: 201-6.

19. Staber PB, Linkesch W, Zauner D, Beham-Schmid C, Guelly C, Schauer S, et al. Common alterations in gene expression and increased proliferation in recurrent acute myeloid leukemia. Oncogene. 2004 : 23: 894-904.

20. Laimer D, Dolznig H, Kollmann K, Vesely PW, Schlederer M, Merkel O, et al. PDGFR blockade is a rational and effective therapy for NPM-ALK-driven lymphomas. Nat Med. 2012; 18: 1699-704.

21. Hui B, Ji H, Xu Y, Wang J, Ma Z, Zhang C, et al. RREB1-induced upregulation of the IncRNA AGAP2-AS1 regulates the proliferation and migration of pancreatic cancer partly through suppressing ANKRD1 and ANGPTL4. Cell Death Dis. 2019; 10: 207

22. Zheng Y, Lu S, Xu Y, Zheng J. Long non-coding RNA AGAP2-AS1 promotes the proliferation of glioma cells by sponging miR-15a/b-5p to upregulate the expression of HDGF and activating Wnt/ beta-catenin signaling pathway. Int J Biol Macromol. 2019; 128: 521-30.

23. Dong H, Wang W, Mo S, Chen R, Zou K, Han J, et al. SP1-induced lncRNA AGAP2-AS1 expression promotes chemoresistance of breast cancer by epigenetic regulation of MyD88. J Exp Clin Cancer Res. 2018; 37: 202

24. Yu W, Gius D, Onyango P, Muldoon-Jacobs K, Karp J, Feinberg AP, et al. Epigenetic silencing of tumour suppressor gene p15 by its antisense RNA. Nature 2008; 451: 202-6. 
25. Gazy I, Zeevi DA, Renbaum P, Zeligson S, Eini L, Bashari D, et al. TODRA, a IncRNA at the RAD51 locus, is oppositely regulated to RAD51, and enhances RAD51-dependent DSB (double strand break) repair. PLoS One. 2015; 10: e0134120.

26. Ahn JY, Rong R, Kroll TG, Van Meir EG, Snyder SH, Ye K. PIKE (phosphatidylinositol 3-kinase enhancer)-A GTPase stimulates Akt activity and mediates cellular invasion. J Biol Chem. 2004; 279: 16441-51.

27. Cai Y, Wang J, Li R, Ayala G, Ittmann M, Liu M. GGAP2/PIKE-a directly activates both the Akt and nuclear factor-kappaB pathways and promotes prostate cancer progression. Cancer Res. 2009; 69: 819-27.

28. He K, Jang SW, Joshi J, Yoo MH, Ye K. Akt-phosphorylated PIKE-A inhibits UNC5B-induced apoptosis in cancer cell lines in a p53-dependent manner. Mol Biol Cell. 2011; 22: 1943-54.

29. Zhang S, Qi Q, Chan CB, Zhou W, Chen J, Luo HR, et al. Fyn-phosphorylated PIKE-A binds and inhibits AMPK signaling, blocking its tumor suppressive activity. Cell Death Differ. 2016; 23: 52-63.

30. Ahn JY, Hu Y, Kroll TG, Allard P, Ye K. PIKE-A is amplified in human cancers and prevents apoptosis by up-regulating Akt. Proc Natl Acad Sci U S A. 2004; 101: 6993-8.

31. Qi Q, He K, Liu X, Pham C, Meyerkord C, Fu H, et al. Disrupting the PIKE-A/Akt interaction inhibits glioblastoma cell survival, migration, invasion and colony formation. Oncogene. 2013; 32: 1030-40.

32. Kim J, Piao HL, Kim BJ, Yao F, Han Z, Wang Y, et al. Long noncoding RNA MALAT1 suppresses breast cancer metastasis. Nat Genet. 2018; 50: 1705-15. 